\title{
Understanding formic acid oxidation mechanism on platinum single crystal electrodes.
}

\author{
Enrique Herrero* and Juan M. Feliu \\ Instituto de Electroquímica, Universidad de Alicante \\ Apdo. 99, E-03080 Alicante, Spain \\ e-mail: herrero@ua.es
}

\begin{abstract}
Recent results on the formic acid oxidation on platinum electrodes, especially those obtained for platinum single crystal electrodes are reviewed. With all the available data, a detailed mechanism for both reaction paths of the mechanism is proposed. For the $\mathrm{CO}$ formation path, oxidation and reduction steps are involved sequentially. On the other hand, the active species is a monodentate adsorbed formate. This adsorption mode is favored in the presence of additional adsorbed species in the neighborhood. The proposed mechanism agrees with all the experimental results obtained for the reaction.
\end{abstract}

\section{Introduction}

Formic acid has been always regarded as a promising reactant for the fuel cell technology. From all the organic molecules susceptible to be used, formic acid is the smallest one and the number of exchanged electrons is only two. For this reason, it has been also regarded as a model molecule in the study of the oxidation of other organic molecules with a more complex structure and oxidation mechanism. Although many efforts have been devoted to the study of the oxidation of these molecules, the overpotentials for the oxidation reaction are still too high for massive practical applications. Thus, better electrocatalyst with lower overpotentials should be developed so that the final energy efficiency of the fuel cell system is maximized. In the research of better electrocatalysts, a crucial piece of information is the 
reaction mechanism. Knowing the details of the reaction mechanism will allow developing new electrode materials which have the property of accelerating the slowest steps in the reaction, in order to solve the bottlenecks in the reaction and to obtain higher efficiencies.

In principle, formic acid oxidation reaction (FAOR) appears to be a reasonably simple reaction since only two electrons are exchanged. In fact, the complete oxidation to $\mathrm{CO}_{2}$ only requires the cleavage of two bonds: an $\mathrm{O}-\mathrm{H}$ bond (which is implied in an acid-base equilibrium) and a $\mathrm{H}-\mathrm{C}$ bond. This second cleavage is probably the most difficult step, but there are pure metals, such as platinum or palladium which are active for this step, due to their excellent properties for hydrogen adsorption. However, when this reaction was first studied on platinum, it was found that a poisoning species [1], which was later identified as adsorbed CO [2], was formed. The formation of $\mathrm{CO}$ implies the presence of unexpected complications in the oxidation mechanism. Moreover, adsorbed CO is oxidized at high potentials, so that it blocks the surface for the reaction. Thus, the oxidation mechanism contains two parallel paths $[3,4]$, one giving rise to $\mathrm{CO}_{2}$ at reasonably low overpotentials through the so-called active intermediate and a chemical dehydration step leading to adsorbed $\mathrm{CO}$, which will be further oxidized to $\mathrm{CO}_{2}$ at higher potentials. Studies with single crystal electrodes have demonstrated that both paths are structure sensitive $[5,6]$. As aforementioned, if the electrocatalysis is to be improved, the key elements in the mechanism should be fully understood. In this manuscript, the recent advances in the knowledge of the oxidation mechanism on platinum electrodes will be reviewed, focusing on the results obtained on well-defined platinum surfaces. The use of well-defined platinum electrodes simplifies the experimental response controlling the surface structure of the electrode allows establishing a clear relationship between surface structure, adsorbed species, and reactivity. These relationships are vital if the oxidation mechanism needs to be solved so that better electrocatalysts are to be proposed from a rational perspective. 


\section{The poisoning path.}

The identification of $\mathrm{CO}$ as the poison intermediate and its role in the oxidation mechanism was possible by DEMS and in situ FTIR [7,8], experiments which serve to experimentally corroborate the existence of a dual pathway. In the recent years, the efforts have been focused to understand the potential and surface structure dependence of the CO formation rate using single crystal electrodes. From the initial studies, it was clear that the CO formation rate on the $\mathrm{Pt}(111)$ was significantly lower than those measured on the other basal planes, namely $\mathrm{Pt}(100)$ and $\mathrm{Pt}(110)[9,10]$. However, until recently, it was not proven that the observed activity of the Pt(111) electrode was due to the presence of surface defects [11-14]. Thus, the use of stepped surfaces with (111) terraces demonstrated that the activity of the ideal (111) plane of the Pt(111) surface was negligible in comparison with that of the steps present on the (111) terrace and also with the (100) surface. Moreover, the turnover rates for the different sites are compared, it has been shown that the activity of the (110) step on the (111) terrace and that of the (100) plane are very similar, and that corresponding to the $\mathrm{Pt}(111)$ is even lower than that expected [15].

Regarding the potential dependence of the reaction rates for $\mathrm{CO}$ formation, it was observed that $\mathrm{CO}$ was formed in a very narrow region (ca. $100 \mathrm{mV}$ wide) $[12,13]$. The center of this region, that is, where the activity for $\mathrm{CO}$ formation reaches the maximum, depends on the surface site and is very close to the potential of zero total charge of the site $[12,13]$. On practical surfaces, that is, platinum nanoparticles, the observed behavior followed the same trends than those obtained for single crystal electrodes, with several local maxima for CO formation, which are associated to the different sites necessarily present on the surface of the nanoparticles [14]. On platinum, the potential of zero total charge (pztc) roughly corresponds with the potential at which the amount of anion and hydrogen species adsorbed is small and balances. In this case, $\mathrm{OH}$ or specifically adsorbed anions are present on the surface at potentials more positive than the pztc, whereas hydrogen is adsorbed at negative values. 
To understand these results, the elementary steps giving rise to the formation of $\mathrm{CO}$ should be analyzed. First of all, the formation of $\mathrm{CO}$ is a more complex reaction than the direct oxidation of formic acid, because it requires the cleavage of the $\mathrm{C}-\mathrm{H}$ and $\mathrm{C}-\mathrm{OH}$ bonds. When compared to the direct oxidation path, the cleavage of this latter bond is significantly more difficult than that of the $\mathrm{O}-\mathrm{H}$ bond. Recent DFT results have shown that the catalytic cleavage of these bonds implies the formation of adsorbed $\mathrm{H}$ and $\mathrm{OH}$ as intermediate species [15]. The formation of these species as intermediates explain the narrow potential window for $\mathrm{CO}$ formation and the inactivity of the (111) surface. If we assume the formation of adsorbed hydrogen and $\mathrm{OH}$, the formation of $\mathrm{CO}$ would then require the simultaneous adsorption of both species. For the $\mathrm{Pt}(111)$ surface, hydrogen and $\mathrm{OH}$ adsorption take place in non-overlapping potential regions, so that, when hydrogen as readily adsorbed, adsorbed $\mathrm{OH}$ is very unstable, and vice versa. Therefore, the reaction rate is negligible for this surface. For $\mathrm{Pt}(100), \mathrm{Pt}(110)$ and step sites, the pztc is located where hydrogen and $\mathrm{OH}$ adsorption regions overlap and $\mathrm{OH}$ and $\mathrm{H}$ coverages are almost identical. Thus, this is the ideal potential in which the reaction should take place, explaining the observed results.

This qualitative hypothesis has been confirmed numerically by potential dependent DFT calculations [15]. Using adsorbed formate species in monodentate configuration as the starting point for the reaction, a detailed mechanism has been proposed (figure 1). As can be seen, initial steps ( $A$ to $D$ in figure 1 ) involve a dehydrogenation process to form a bidentate adsorbed carboxylate (figure 1D), whereas in the second part of the mechanism ( $\mathrm{D}$ to $\mathrm{H}$ in figure 1 ), the $\mathrm{C}-\mathrm{OH}$ bond is broken to yield adsorbed $\mathrm{CO}$ and $\mathrm{OH}$ (figure 1G), which is eventually desorbed as $\mathrm{OH}^{-}$(figure $1 \mathrm{H}$ ). The energetics for this mechanism at different electrode potential is displayed in figure 2. As can be seen, the $\mathrm{Pt}(111)$ surface has the highest activation energy for the reaction as compared with a stepped surface or the $\mathrm{Pt}(100)$ electrode, in good agreement with the experimental results. Since the initial steps involve the formation of adsorbed hydrogen and its desorption in an oxidation step, this part of the mechanism is favored as the potential is made 
more positive, as can be observed in figures $2 A-C$. However, the final steps ( $D$ to $H$ ) become less favorable as the potential increases since the final step which involves the desorption of $\mathrm{OH}$ is a reductive process. As can be seen in figure 2 , these steps become uphill at $0.6 \mathrm{~V}$ for the $\mathrm{Pt}(100)$ and $\mathrm{Pt}(553)$ surface since $\mathrm{D}$ state has lower energy than that of the final $\mathrm{H}$ state. For the $\mathrm{Pt}(100)$ and stepped surfaces, a compromise between both trends is obtained at ca. $0.3 \mathrm{~V}$, where the global activation barrier for the process reaches a minimum. On the other hand, for the $\mathrm{Pt}(111)$ electrode, the barriers, especially that related to the cleavage of the $\mathrm{C}-\mathrm{H}$ bond (step $\mathrm{B}$ ), are still very high in the whole potential range, so that measurable reaction rates would be negligible. This detailed scheme is also in agreement with additional experimental pieces of evidence: i) Electrochemical and spectroscopic results implied a general mechanism for the CO formation path involving, sequentially, oxidation and reduction steps $[16,17]$ and ii) it has been shown that this reaction path requires more than 2 neighboring free Pt sites to occur $[18,19]$. As can be observed in figure 1 , the formation of $\mathrm{CO}$ requires several contiguous free sites.

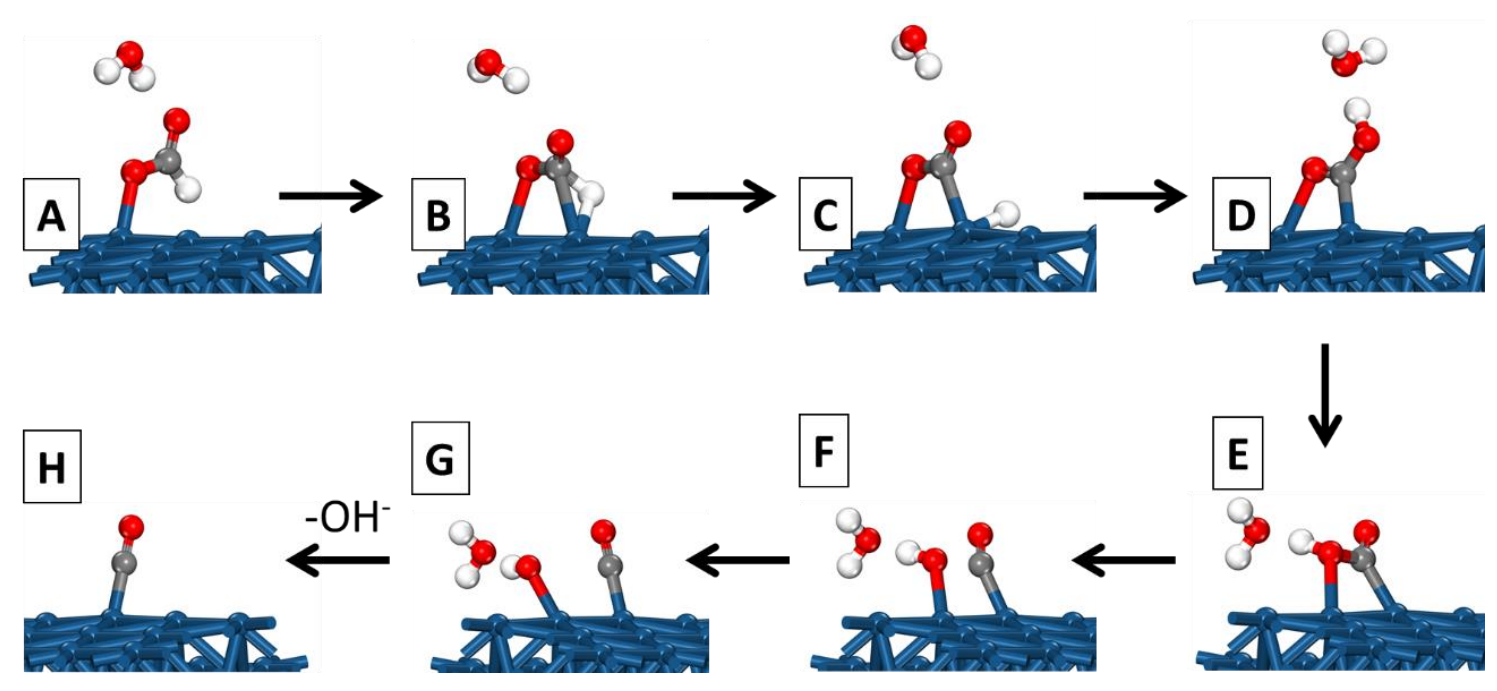

Figure 1. Reaction scheme for the formation of $\mathrm{CO}$ on platinum from monodentate adsorbed formate. In this scheme, A, C, D, G, and H are stable states, E is a metastable state and B and F are transition states. Adapted from reference [15]. 


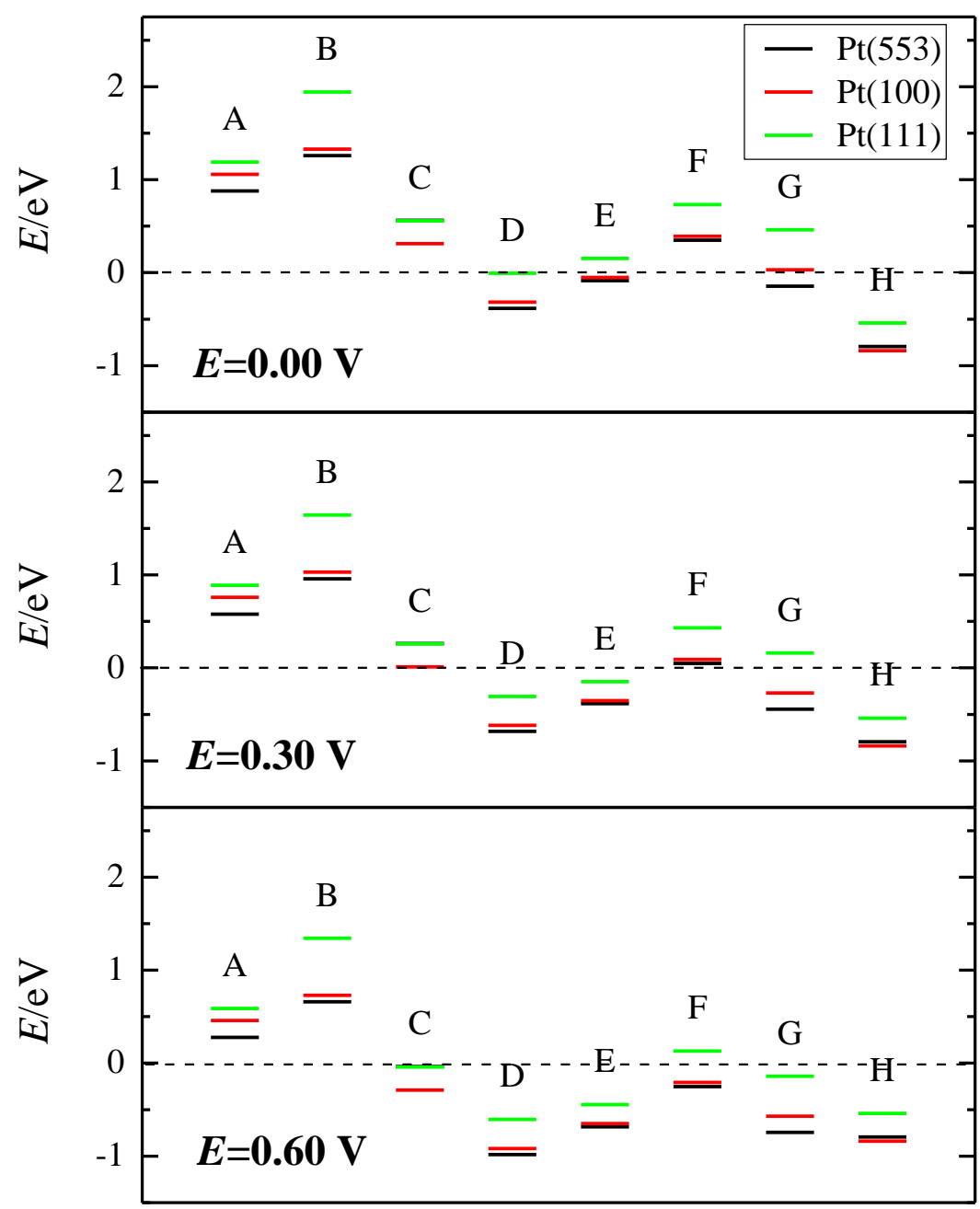

Figure 2. Energetics of the states, pseudo-states, and transition states involved in the proposed $\mathrm{CO}$ formation mechanism (see figure 1), estimated on different platinum surfaces different electrode potentials vs. RHE.

\section{The active intermediate path.}

As aforementioned, the desired path for the reaction is that taking place through the active intermediate. However, the reactivity is significantly affected by the CO formation path. To put this effect into perspective, the reaction rates for both paths should be compared. It has been found that the maximum rates for the active intermediate path are at least two orders of magnitude higher than those measured for the CO formation steps [15]. This implies that, in the global mechanism, the $\mathrm{CO}$ formation path is a minor side path which has a significant effect on the overall reactivity, because its product accumulates on the surface. This fact highlights the importance of controlling every single detail of the reaction mechanism. 
To gain a detailed knowledge of the direct path, different reaction conditions and techniques should be explored. Thus, experiments at different $\mathrm{pH}$ values have been conducted to determine the active species in the reaction, since the reaction can be initiated either from bulk formate or formic acid. These studies have shown that currents were proportional to the concentration of solution formate $[15,20-23]$, provided that the measurements are carried out in absence of specific anion adsorption. It has been shown that the specific adsorption of the anions present in the buffered solutions at neutral $\mathrm{pH}$ alters the reactivity of the surface $[22,23]$. These results were interpreted as the active species in the oxidation reaction is solution formate. However, the correct interpretation is that one proton has transferred before the rds. This implies that the reaction can be initiated from $\mathrm{HCOO}^{-}$or if the initial species is formic acid, this species has transferred one proton before the rds [24]. Additionally, using the theory for protonelectron transfer reaction, all these $\mathrm{pH}$ dependent studies reveal that the rate determining step involves a concerted proton-electron transfer. $[25,26]$

IR spectroscopy, especially under Attenuated Total Reflection (ATR) configuration also provided hints on the mechanism, since bidentate adsorbed formate (bonded to the surface by the two oxygen atoms) was detected in the same region where formic acid oxidation reaction takes place. Additionally, specific adsorption of the formate anion was also detected on the $\mathrm{Pt}(111)$ electrodes using fast voltammetry [27]. Although the role played by this species in the mechanism was subject of a long debate, a general consensus has been finally reached nowadays. The analysis of the IR data coupled with analytical detection suggested that this species was not the active intermediate [28]. Recently, a detailed analysis of the spectroscopic data revealed that the measured currents were not proportional to the adsorbate coverage, which reinforced the idea that the adsorbed bidentate formate was not true active intermediate [29]. Also, some DFT calculation revealed that the cleavage of the $\mathrm{C}-\mathrm{H}$ bond in this configuration was unlikely, due to a high activation energy [30]. 
Having discarded bidentate adsorbed formate as the active intermediate, another species should be proposed. Since the most difficult step in the reaction is the cleavage of the $\mathrm{C}-\mathrm{H}$ bond, the reaction should take place from an adsorbed configuration in which this cleavage is feasible. The results with adatom modified surfaces have shown that, when the $\mathrm{C}-\mathrm{H}$ bond is pointing to the surface, the cleavage is almost barrierless due to the affinity of the platinum surface for hydrogen [31,32]. This is the case of monodentate adsorbed formate shown in figure 1A. However, this configuration rapidly evolves to the most stable configuration of adsorbed bidentate formate (figure 3).
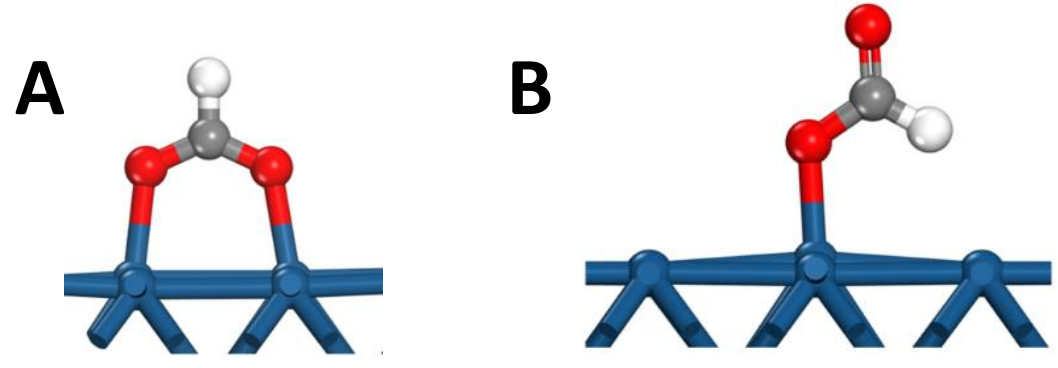

Figure 3. Bidentate $(A)$ and monodentate $(B)$ adsorbed formate on a platinum single crystal. In this configuration, the cleavage of the $\mathrm{C}-\mathrm{H}$ bond is facile, due to the interaction of the $\mathrm{H}$ atom with the surface.

However, bidentate adsorbed formate is not a dead end or undesired species in the mechanism; there are pieces of evidence that adsorbed species and also bidentate formate can have an active role in the mechanism. First, on gold electrodes, the kinetic analysis of this reaction indicates a quadratic dependence on the coverage of the adsorbed species [33]. On the other hand, experiments with different supporting electrolytes indicate that some adsorbed anions, such as sulfate or acetate, catalyze the oxidation of formic acid on platinum electrodes [34]. In general, the presence of adsorbed species would hinder the electrocatalytic activity of the surface, but this effect is not observed on Pt(111) electrodes for formic acid oxidation, where the reaction seems catalyzed by the presence of some adsorbed anions, such as sulfate or 
acetate. In fact, the calculated apparent activation energy reaches a minimum at the onset of the adsorption of these anions. These facts led to propose a reaction model in which the active species is monodentate adsorbed formate, which is able to interact with the surface through the $\mathrm{C}-\mathrm{H}$ bond in a site adjacent to a bidentate adsorbed formate (or another anion such as sulfate or acetate). The resulting kinetic model was able to reproduce the measured currents for the Pt(111) electrode [34].

These results have been confirmed by DFT calculations [15]. In the monodentate configuration, the adsorbed species can rotate freely through the $\mathrm{O}-\mathrm{C}$ bond until the second oxygen bond comes close to the surface, giving rise immediately to the bidentate form. However, this rotation is precluded when the monodentate species is surrounded by bidentate species forming a pocket (figure 4). In this pocket, the formation of the bidentate form is inhibited and the cleavage of the $\mathrm{C}-\mathrm{H}$ can take place. This mechanism also explains two experimental facts: the different reactivity of the surfaces and the inhibition of the reactivity at potentials above $0.8 \mathrm{~V}$ vs. RHE. In the first case, measured currents for the Pt(100) electrode are more than one order of magnitude higher than those measured for the Pt(111) electrode. Since the energetics of the $\mathrm{C}-\mathrm{H}$ bond cleavage is very similar for the $\mathrm{Pt}(100)$ and $\mathrm{Pt}(111)$ electrode, the difference in the currents should be then related to the adsorption of anions that facilitate the stabilization of the monodentate form. On the Pt(100) electrode, the adsorption process of the anions is faster and takes place in a narrower potential window, explaining the higher currents. On the other hand, when the anion adlayer has reached the maximum packing density, irrespectively of the substrate structure, there are no free pockets available for the adsorption of monodentate adsorbed formate, and thus, currents are negligible at high potentials. 


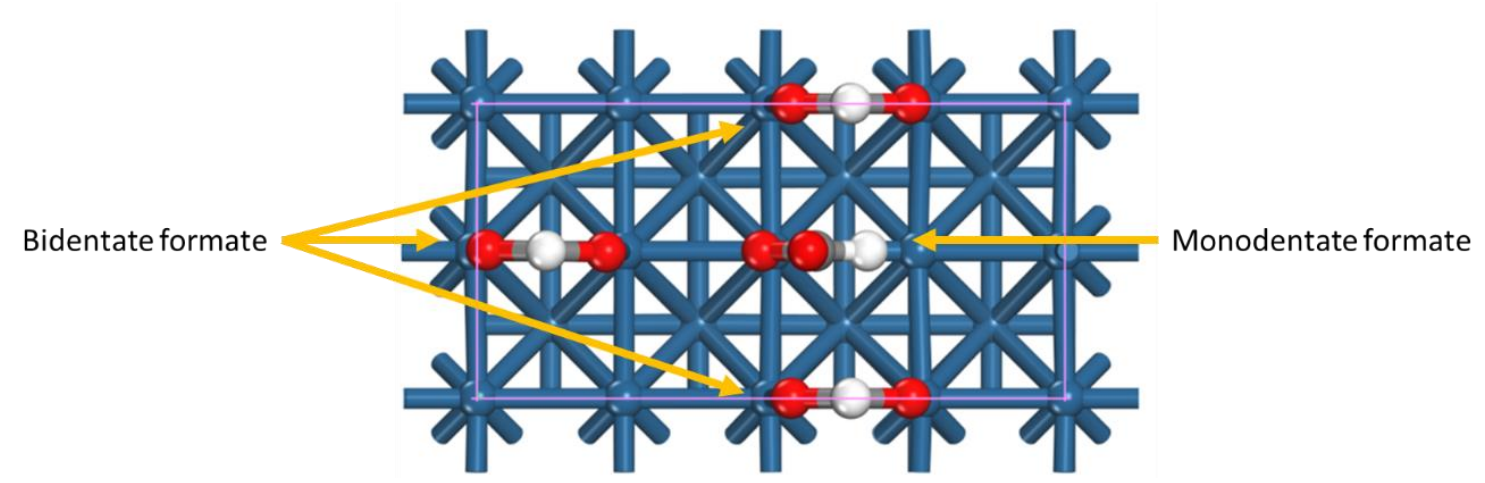

Figure 4. Monodentate adsorbed formate inside a pocket of preadsorbed bidentate formate on the $\mathrm{Pt}(100)$ surface. In this configuration, monodentate formate cannot rotate to yield the bidentate configuration. Adapted from reference [15].

As a summary, a detailed mechanism for the formic acid oxidation reaction has been described. In this mechanism, the active intermediate is the monodentate adsorbed formate. However, on platinum, this species can either react to yield $\mathrm{CO}_{2}$ or transform into the most stable adsorbed form: the bidentate configuration. The undesired transition is hindered by the presence of additional adsorbed species, which facilitates the reactivity of the active intermediate. Thus, the key to activate the reactivity for this process is to hinder the transformation to the bidentate form or to improve the energetics of the monodentate form with respect to the bidentate one. On the other hand, the formation of $\mathrm{CO}$, which is an undesired and poisoning intermediate, requires that $\mathrm{OH}$ and $\mathrm{H}$ adsorption processes could take place simultaneously at the same electrode potential.

\section{Acknowledgments.}

This work has been financially supported by the MINECO (Spain) and Generalitat Valenciana through projects CTQ2016-76221-P (AEI/FEDER, UE) and PROMETEOII/2014/013, respectively. 
References and recommended reading.

1. Capon A, Parsons R: The oxidation of formic acid at noble metal electrodes Part III. Intermediates and mechanism on platinum electrodes J. Electroanal. Chem. 1973, 45:205-231.

2. Beden B, Bewick A, Lamy C: A comparative study of formic acid adsorption on a platinum electrode by both electrochemical and emirs techniques J. Electroanal. Chem. 1983, 150:505-511.

3. Parsons R, Vandernoot $\mathrm{T}$ : The oxidation of small organic molecules: A survey of recent fuel cell related research. J. Electroanal. Chem. 1988, 257:9-45.

4. Koper MTM, Lai SCS, Herrero E: Mechanisms of the Oxidation of Carbon Monoxide and Small Organic Molecules at Metal Electrodes. In Fuel Cell Catalysis, A Surface Science Approach. Edited by Koper MTM: John Wiley \& Sons, Inc; 2009:159-208.

5. Clavilier J, Parsons R, Durand R, Lamy C, Leger JM: Formic acid oxidation on single crystal platinum electrodes. Comparison with polycrystalline platinum. J. Electroanal. Chem. 1981, 124:321-326.

6. Adzic RR, Tripkovic AV, O'Grady WE: Structural effects in electrocatalysis. Nature 1982, 296:137-138.

7. Wolter O, Willsau J, Heitbaum J: Reaction Pathways of The Anodic Oxidation of Formic Acid on Platinum Evidenced by Oxygen-18 Labeling-A DEMS Study. Journal of Electrochemical Society 1985, 132:1635-1638.

8. Sun SG, Clavilier J, Bewick A: The mechanism of electrocatalytic oxidation of formic acid on Pt (100) and Pt (111) in sulphuric acid solution: an emirs study. J. Electroanal. Chem. 1988, 240:147-159.

9. Herrero E, Fernández-Vega A, Feliu JM, Aldaz A: Poison formation reaction from formic acid and methanol on $\mathrm{Pt}(\mathbf{1 1 1})$ electrodes modified by irreversibly adsorbed $\mathrm{Bi}$ and $\mathrm{As}$ J. Electroanal. Chem. 1993, 350:73-88.

10. Herrero E, Feliu JM, Aldaz A: Poison formation reaction from formic acid on $\mathrm{Pt}(\mathbf{1 0 0})$ electrodes modified by irreversibly adsorbed bismuth and antimony J. Electroanal. Chem. 1994, 368:101-108.

11. Maciá MD, Herrero E, Feliu JM, Aldaz A: Formic acid self-poisoning on bismuthmodified stepped electrodes. J. Electroanal. Chem. 2001, 500:498-509.

12. * Grozovski V, Climent V, Herrero E, Feliu JM: Intrinsic Activity and Poisoning Rate for $\mathrm{HCOOH}$ Oxidation at Pt(100) and Vicinal Surfaces Containing Monoatomic (111) Steps. ChemPhysChem 2009, 10:1922-1926.

The rates for the direct path and the CO formation path are given, finding that the maximum rate is at the pztc.

13. * Grozovski V, Climent V, Herrero E, Feliu JM: Intrinsic activity and poisoning rate for $\mathrm{HCOOH}$ oxidation on platinum stepped surfaces. Phys. Chem. Chem. Phys. 2010, 12:8822-8831.

The rates for the direct and the $\mathrm{CO}$ formation path on different surfaces are given and analyzed.

14. Grozovski V, Solla-Gullon J, Climent V, Herrero E, Feliu JM: Formic Acid Oxidation on Shape-Controlled Pt Nanoparticles Studied by Pulsed Voltammetry. J. Phys. Chem. C 2010, 114:13802-13812.

15. $\quad{ }^{* *}$ Ferre-Vilaplana A, Perales-Rondon JV, Buso-Rogero C, Feliu JM, Herrero E: Formic acid oxidation on platinum electrodes: a detailed mechanism supported by experiments and calculations on well-defined surfaces. J. Mater. Chem. A 2017, 5:21773-21784.

DFT studies on the reaction providing a detailed mechanism for the direct and CO formation paths.

16. Lu GQ, Crown A, Wieckowski A: Formic acid decomposition on polycrystalline platinum and palladized platinum electrodes. J. Phys. Chem. B 1999, 103:9700-9711. 
17. Cuesta A, Cabello G, Gutierrez C, Osawa M: Adsorbed formate: the key intermediate in the oxidation of formic acid on platinum electrodes. Phys. Chem. Chem. Phys. 2011, 13:20091-20095.

18. Leiva E, Iwasita T, Herrero E, Feliu JM: Effect of adatoms in the electrocatalysis of HCOOH oxidation. A theoretical model. Langmuir 1997, 13:6287-6293.

19. Cuesta A, Escudero Ma, Lanova B, Baltruschat H: Cyclic Voltammetry, FTIRS, and DEMS Study of the Electrooxidation of Carbon Monoxide, Formic Acid, and Methanol on Cyanide-Modified Pt(111) Electrodes. Langmuir 2009, 25:6500-6507.

20. **Joo J, Uchida T, Cuesta A, Koper MTM, Osawa M: Importance of Acid-Base Equilibrium in Electrocatalytic Oxidation of Formic Acid on Platinum. J. Am. Chem. Soc. 2013, 135:9991-9994.

The effects of $\mathrm{pH}$ in the reaction are studied on polycrystallyne electrodes.

21. Joo J, Uchida T, Cuesta A, Koper MTM, Osawa M: The effect of pH on the electrocatalytic oxidation of formic acid/formate on platinum: A mechanistic study by surfaceenhanced infrared spectroscopy coupled with cyclic voltammetry. Electrochim. Acta 2014, 129:127-136.

22. Brimaud S, Solla-Gullon J, Weber I, Feliu JM, Behm RJ: Formic Acid Electrooxidation on Noble-Metal Electrodes: Role and Mechanistic Implications of pH, Surface Structure, and Anion Adsorption. ChemElectroChem 2014, 1:1075-1083.

23. **Perales-Rondón JV, Brimaud S, Solla-Gullón J, Herrero E, Jürgen Behm R, Feliu JM: Further Insights into the Formic Acid Oxidation Mechanism on Platinum: pH and Anion Adsorption Effects. Electrochim. Acta 2015, 180:479-485.

The effects of $\mathrm{pH}$ and anion adsoprtion on the reaction are analyzed.

24. Wei Y, Zuo XQ, He ZD, Chen W, Lin CH, Cai J, Sartin M, Chen Y-X: The mechanisms of $\mathrm{HCOOH} / \mathrm{HCOO}-$ oxidation on Pt electrodes: Implication from the pH effect and H/D kinetic isotope effect. Electrochem. Commun. 2017, 81:1-4.

25. **Koper MTM: Theory of multiple proton-electron transfer reactions and its implications for electrocatalysis. Chem. Sci. 2013, 4:2710-2723.

The theory for pronton-coupled transfer reactions is formulated

26. ${ }^{* *}$ Koper MTM: Volcano Activity Relationships for Proton-Coupled Electron Transfer Reactions in Electrocatalysis. Top. Catal. 2015, 58:1153-1158.

It stablishes how proton-coupled electron transfer reactions affects the electrocatalysis

27. Grozovski V, Vidal-Iglesias FJ, Herrero E, Feliu JM: Adsorption of Formate and Its Role as Intermediate in Formic Acid Oxidation on Platinum Electrodes. ChemPhysChem 2011, 12:1641-1644.

28. ${ }^{*}$ Chen YX, Heinen M, Jusys Z, Behm RJ: Bridge-bonded formate: Active intermediate or spectator species in formic acid oxidation on a Pt film electrode? Langmuir 2006, 22:10399-10408.

It is proposed that bridged bonded formate is not the active intermidiate.

29. ${ }^{* *} X u$ J, Yuan DF, Yang F, Mei D, Zhang ZB, Chen YX: On the mechanism of the direct pathway for formic acid oxidation at a Pt(111) electrode. Phys. Chem. Chem. Phys. 2013, 15:4367-4376.

It stablishes that bridged bonded formate is not the active intermediate using experimental data.

30. **Wang H-F, Liu Z-P: Formic Acid Oxidation at Pt/H2O Interface from Periodic DFT Calculations Integrated with a Continuum Solvation Model. J. Phys. Chem. C 2009, 113:17502-17508.

DFT studies on the reaction highlighting the importance of solvation water and adsorption configuration.

31. *Perales-Rondon JV, Ferre-Vilaplana A, Feliu JM, Herrero E: Oxidation Mechanism of Formic Acid on the Bismuth Adatom-Modified Pt(111) Surface. J. Am. Chem. Soc. 2014, 136:13110-13113. 
It stablishes the mechanism of bismtuh catalysis in the reaction.

32. *Ferre-Vilaplana A, Perales-Rondon JV, Feliu JM, Herrero E: Understanding the Effect of the Adatoms in the Formic Acid Oxidation Mechanism on Pt(111) Electrodes. ACS Catal. 2015, 5:645-654.

It relates the electronegativity of the adatom with is catalityc effec, using expeimental and DFT calculations.

33. Cuesta A, Cabello G, Hartl FW, Escudero-Escribano M, Vaz-Domínguez C, Kibler LA, Osawa M, Gutiérrez C: Electrooxidation of formic acid on gold: An ATR-SEIRAS study of the role of adsorbed formate. Catal. Today 2013, 202:79-86.

34. *Perales-Rondon JV, Herrero E, Feliu JM: Effects of the anion adsorption and pH on the formic acid oxidation reaction on Pt(111) electrodes. Electrochim. Acta 2014, 140:511517.

The effects of $\mathrm{pH}$ and anion adsorption in the reaction are analyzed. 
